\title{
Adaptabilidad y potencial agronómico de 12 cultivares de tomate (Lycopersicum esculentum Mill) en condiciones de casa malla, Managua, Nicaragua
}

\section{Adaptability and agronomic potential of 12 tomato cultivars (Lycopersicum esculentum Mill) under protected conditions Managua, Nicaragua}

\author{
Jorge Gómez-Martínez ${ }^{1}$ Vidal Marín Fernández ${ }^{2}$, Delia María Gómez Peralta ${ }^{3}$, Evert Francisco Herrera Fuentes ${ }^{3}$ \\ ${ }^{1}$ MSc. en fitomejoramiento, ${ }^{2}$ MSc. en agroecología y desarrollo sostenible, ${ }^{3}$ Ing. agrónomo Universidad Nacional Agraria.
}

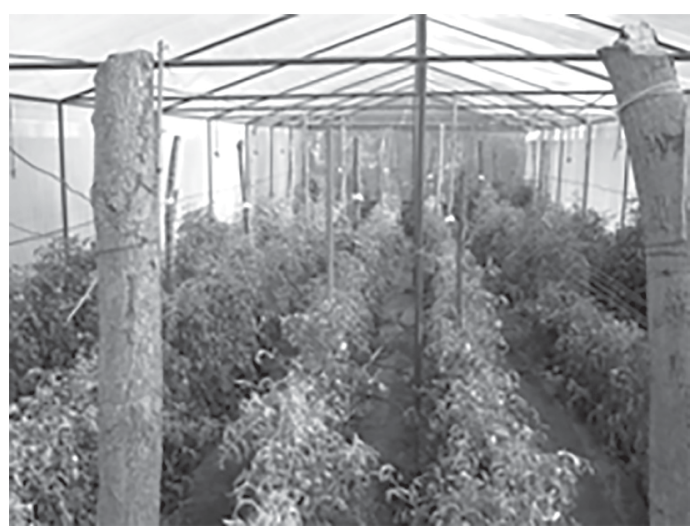

\section{RESUMEN}

El uso de casas malla en la producción de tomate permite un aumento en los rendimientos y la calidad del fruto. El objetivo de este estudio fue generar información sobre el comportamiento agronómico de 12 cultivares de tomate (Solanum lycopersicum Mill) producidos en condiciones de casa malla, trabajo realizado en el Centro de Experimentación y Validación de Tecnología (CEVT) Las Mercedes, en la Universidad Nacional Agraria (UNA) en Managua, Nicaragua. Se utilizó un diseño experimental en Látice rectangular de 3x4 (tres réplicas y cuatro surcos) repetidos en dos bloques. Los datos se analizaron a través de un análisis de varianza y diferencia mínima significativa. Se registró la información de diez variables basadas en el comportamiento agronómico, siete cuantitativas (número de frutos, peso de fruto, rendimiento, número de lóculos, diámetro polar y ecuatorial, grados brix) y tres cualitativa (color e intensidad del fruto maduro y forma del fruto). Los resultados indican que los genotipos mostraron un comportamiento similar respecto a las variables: diámetro ecuatorial, diámetro polar, grados brix y número de lóculos. Los cultivares Shanty, CLN3125L, AVTO1032 y AVTO1059 registraron los mayores rendimientos. En base al análisis de comportamiento agronómico los genotipos promisorios más destacados para continuar con evaluaciones más exigente en condiciones de casa malla son: CLN3125L, AVTO1032, AVTO1059, AVTO1058, AVTO1078, AVTO1005, AVTO1008 y 1x10.

Palabras clave: genotipo, látice, agricultura protegida, comportamiento agronómico.

\section{ABSTRACT}

This study was carried out in the Center for Experimentation and Validation of Technology (CEVT) Las Mercedes UNA Located in Managua; the purpose of this study was to generate information on the agronomic performance of 12 cultivars of tomato (Lycopersicum esculentum Mill) produced in net house conditions. The experimental design used was a $3 \times 4 \mathrm{~m}$ rectangular lattice with two repetitions. The data was evaluated through analysis of variance ANOVA and LSD 95\% of confident. The information of ten fruit characters was recorded, seven of them were quantitative and three rest qualitative. The analysis showed that the genotypes evaluated in open field conditions and in greenhouse conditions presented a similar behavior in the case of: polar and equatorial diameter, brix degrees, and number of cores. Whereas the Shanty genotypes CLN3125L, AVTO1032y AVTO1059 gave the highest yields in the greenhouse. Based on the analysis of agronomic performance, the promising genotypes to continue AVTO1032, AVTO1059, AVTO1058, AVTO1078, AVTO1005, AVTO1008 and 1x10).

Keywords: Genotype, lattice, protected agriculture, agronomic behavior. 
$\mathrm{E}$ 1 tomate (Lycopersicum esculentum Mill) es originario de los Andes (Perú, Bolivia y Ecuador), región en la que se puede encontrar gran cantidad de variedades silvestres (Smith, 1994). Sin embargo, (Jaramillo et al., 2006), afirman que aunque es originario de América del Sur, entre las regiones de Chile, Ecuador y Colombia, su domesticación se realizó en el sur de México y norte de Guatemala.

El tomate es uno de las hortalizas más importante del mundo y su popularidad aumenta constantemente. En el ámbito mundial, se clasifica como el segundo vegetal más importante, superado únicamente por la papa (Solanum tuberosum L.) (INTA, 2012).

Los principales países productores son China, Estados Unidos, Turquía, Egipto, Italia, India, Irán, España, Brasil y México, los cuales contribuyen con cerca del 70\% de la producción mundial (Jaramillo, 2006). En Centroamérica los mayores productores de tomate son Guatemala, Honduras y Costa Rica (EDA, 2006).

El tomate se cultiva en Nicaragua desde la década de 1940, iniciándose en el municipio de Tisma, departamento de Masaya y posteriormente en el resto del país (Rayo, 2001).

Las principales áreas de producción de tomate en Nicaragua, están ubicadas en los departamentos de Matagalpa y Jinotega, particularmente en el Valle de Sébaco y Tomatoya. También se produce en menor escala en las zonas de Estelí, Malacatoya, Tisma y Nandaime (MAGFOR, 2012a).

El sistema de producción de tomate bajo condiciones protegidas es relativamente nuevo en el país, generando un impacto importante en los últimos años, por su incremento en área, productividad, rentabilidad y calidad del producto.

La producción de tomate en condiciones de casa malla o ambiente protegido permite incrementar el rendimiento, calidad de frutos y genera mayor beneficio económico. Este sistema permite cosechar fuera de época, mejor calidad del fruto, preservación de la estructura del suelo, siembra de materiales seleccionados, aumento considerable de la producción, ahorro en los costos de producción y disminución en la utilización de pesticidas (Jaramillo et al., 2007).

Este sistema de producción se caracteriza por la protección mediante estructuras verticales y cobertura de plástico, con el fin de evitar el impacto de la lluvia sobre el cultivo $\mathrm{y}$ ataque de plagas. Su manejo tecnológico es igual al que tradicionalmente se le hace al cultivo de tomate a libre exposición, sin embargo, surge la necesidad de introducir nuevos cultivares que garanticen al productor, optimizar la producción y generar buena rentabilidad en este tipo de sistema de producción.

La producción de tomate a campo abierto resulta cada vez más difícil, debido a condiciones ambientales adversas, resistencia e incidencia de plagas y enfermedades que afectan la productividad de este cultivo (Carrillo et al., 2003).
En nuestro país una de las principales limitantes es el limitado material genético para este tipo de sistema de producción y la poca información que existe en cuanto a los rendimientos que se producen. Sin embargo, en los últimos años se han desarrollado programas de mejoramiento de variedades que se adaptan a diferentes ambientes como en estructuras cerradas, el Asían Vegetable Research and Development Center (AVRDC) cada año desarrolla nuevas líneas, las cuales distribuye a varios países del mundo para que sean evaluadas y seleccionadas bajo las presiones de las diferentes condiciones ambientales en las localidades de cada país.

Según (Jaramillo et al., 2006) el rendimiento promedio obtenido en casas malla es entre 5 y $8 \mathrm{~kg}_{\text {planta }}{ }^{-1}$, superando tres veces el que se obtiene a libre exposición, que está entre 1.5 y $2 \mathrm{~kg}$ planta $^{-1}$. En países como México, el rendimiento de tomate en ambientes protegidos es de 150 a $200 \mathrm{t}$ $\mathrm{ha}^{-1}$, mientras que en los EE.UU. y Canadá se alcanzan rendimientos de hasta $450 \mathrm{t} \mathrm{ha}^{-1}$ (López et al., 2012). En Nicaragua existen aproximadamente 20 hectáreas sembradas bajo este tipo de sistema de cultivo, sin embargo, no se conoce con exactitud los rendimientos que se obtienen.

Ante esta problemática, se plantea la necesidad de evaluar el potencial del rendimiento agronómico de doce líneas de tomate y su adaptabilidad bajo ambiente protegido (casa malla); once de ellas procedentes del centro internacional de hortalizas de Taiwán y un hibrido que se cultivan actualmente en nuestro país.

\section{MATERIALES Y MÉTODOS}

Ubicación del ensayo. Se estableció en una de las casas malla ubicada en el Centro de experimentación y validación tecnológica (CEVT) Las Mercedes, propiedad de la Universidad Nacional Agraria y ubicada del km 11 de la carretera Norte en Managua, 800 metros al norte, entre las coordenadas $12^{\circ} 08^{\prime} 05^{\prime \prime}$ de latitud norte y $86^{\circ} 09^{\prime} 22^{\prime \prime}$ de longitud oeste, a una altitud de $56 \mathrm{msnm}$ (INETER, 2013). La casa malla posee una dimensión de $27 \mathrm{~m}$ de largo y ocho metros de ancho, para un área de $216 \mathrm{~m}^{2}$. El trasplante se realizó en la segunda semana del mes de noviembre de 2012, cosechándose en marzo de 2013.

Diseño experimental. El diseño experimental utilizado fue un Látice rectangular 3x4 con doce tratamientos, de los cuales se hicieron dos réplicas. Se trabajó con el hibrido comercial Shanty como testigo. Cada tratamiento consistió de un surco de $2.5 \mathrm{~m}$ lineales, con un espaciamiento entre surco de $0.8 \mathrm{~m}$ y $0.5 \mathrm{~m}$ entre planta, para una parcela útil de $2 \mathrm{~m}^{2}$. Los cultivares se describen en el cuadro 1 . 
Cuadro 1. Descripción de los cultivares de tomate utilizados en el estudio

\begin{tabular}{ccccl}
\hline$N^{\circ}$ & Cultivares & $\begin{array}{c}\text { Composición } \\
\text { genética }\end{array}$ & $\begin{array}{c}\text { Habito de } \\
\text { crecimiento }\end{array}$ & Origen \\
\hline 1 & CLN3125L & Línea & Indeterminado & AVRDC (Taiwán) \\
2 & AVTO1059 & Línea & Indeterminado & AVRDC (Taiwán) \\
3 & AVTO1005 & Línea & Indeterminado & AVRDC (Taiwán) \\
4 & AVTO1004 & Línea & Indeterminado & AVRDC (Taiwán) \\
5 & $1 \times 10$ & Línea & Indeterminado & Wisconsin University \\
6 & AVTO1023 & Línea & Indeterminado & AVRDC (Taiwán) \\
7 & AVTO1058 & Línea & Indeterminado & AVRDC (Taiwán) \\
8 & AVTO1032 & Línea & Indeterminado & AVRDC (Taiwán) \\
9 & AVTO1143 & Línea & Indeterminado & AVRDC (Taiwán) \\
10 & AVTO1008 & Línea & Indeterminado & AVRDC (Taiwán) \\
11 & AVTO1078 & Línea & Indeterminado & AVRDC (Taiwán) \\
12 & Shanty & Hibrido & Semideterminado & Hazera genetics (Israel) \\
\hline
\end{tabular}

AVRDC (Asian Vegetable Research and Development Center).

Variables evaluadas. Número de frutos cosechados por planta, diámetro polar del fruto $(\mathrm{cm})$, diámetro ecuatorial del fruto $(\mathrm{cm})$, peso del fruto $(\mathrm{g})$, rendimiento $\left(\mathrm{kg} \mathrm{ha}^{-1}\right)$, número de lóculos, grados BRIX, color del fruto maduro, intensidad de color del fruto maduro y forma del fruto.

Análisis de datos. Los datos fueron analizados a través de un análisis de varianza y separación de medias por diferencia mínima significativa.

\section{Manejo agronómico}

Establecimiento de semillero. Se estableció en una casa malla ubicada en la Universidad Nacional Agraria, usando bandejas de polietileno de 78 celdas (una semilla por celda), rellenadas con sustrato Kekkila Garden el que se desinfectó con una solución de diez ml de ácido cítrico (Biolife 20SL ${ }^{\circledR}$ ) y cinco $\mathrm{ml}$ de Metilbenzimidazole $\left(\right.$ Carbendazin $\left.{ }^{\circledR}\right)$, ambos en 10 litros de agua. Se regó dos veces al día, en la mañana y en la tarde.

Preparación del terreno. Se prepararon camellones con altura de $30 \mathrm{~cm}$. Estos se realizaron utilizando azadones.

Trasplante. Se realizó a los 28 días después de la germinación cuando las plántulas alcanzaron una altura de 10 y 15 $\mathrm{cm}$, se seleccionaron plántulas sanas y vigorosas que presentaron de cuatro a seis hojas.

Tutoreo y amarre. Se efectuó cuando la planta tenía $25 \mathrm{~cm}$ de altura. Se utilizó el sistema de espaldera, que consistió en colocar varillas de hierro de media pulgada de grosor y tres metros de altura, distanciadas a tres metros y a lo largo de cada surco. Se ubicaron dos hileras de cuerda de nylon con un espaciamiento de $15 \mathrm{~cm}$. El propósito fue sujetar las plantas, evitar el acame y el contacto del follaje y los frutos con el suelo.
Riego. El sistema de riego fue por goteo con frecuencia inicial de 30 minutos durante los primeros 30 días después del trasplante, luego la frecuencia aumentó a 45 minutos, después a una hora al día, hasta llegar a dos horas por día (una por la mañana y otra por la tarde). La cantidad de agua por hora de riego fue igual a 1.6 litros por gotero.

Fertilización. Al momento del trasplante se aplicó como solución arrancadora la fórmula 18-46-00, diluyendo 320 gramos en 30 litros de agua, más una aplicación edáfica de 12-30-10 en dosis de $190 \mathrm{~kg} \mathrm{ha}^{-1}$. La segunda y tercera fertilización edáfica se realizó a los 15 y a los 45 días después del trasplante (ddt), usando en cada momento $180 \mathrm{~kg} \mathrm{ha}^{-1}$ de urea $46 \%$. A partir de los $15 \mathrm{ddt}$ se realizaron aplicaciones foliares cada ocho días y hasta el inicio de la floración, usando foliar mix (15-30-15) más quelato de zinc $(14 \%)$, quelato de calcio $(10 \%)$, quelato de magnesio (7\%), quelato de boro (15\%) en dosis de $285 \mathrm{ml} \mathrm{de}$ cada producto por hectárea. Posteriormente desde el inicio de formación de frutos hasta la cosecha se continuaron las aplicaciones foliares con potasio (36\%), calcio y multifruto ${ }^{\circledR}$ (30\%) en dosis de $712 \mathrm{ml} \mathrm{ha}^{-1}$ en igual intervalo de tiempo.

Manejo fitosanitario. En la etapa de semillero se aplicó Metilbenzimidazole en dosis de cinco $285 \mathrm{ml} \mathrm{ha}^{-1}$ y ácido cítrico en dosis de $2850 \mathrm{ml} \mathrm{ha}^{-1}$. Ambos productos se aplicaron como preventivo para el mal del talluelo (Pythium sp.). Las enfermedades que se presentaron en la etapa vegetativa fueron Tizón Temprano (Alternaria solani) y Botrytis (Botrytis cinerea). El tizón temprano se manejó usando Avante $\left(\right.$ Mancozeb $^{\circledR}$ ) en dosis de $0.43 \mathrm{~kg} \mathrm{ha}^{-1}$; Propiconazole (Molto $49^{\circledR}$ ), Metilbenzimidazole y Fosetil Aluminio (Prevalor ${ }^{\circledR}$ ), todos ellos en dosis de $285 \mathrm{ml} \mathrm{ha}^{-1}$. Para el caso de Botritis también se aplicó Metilbenzimidazole y Sulfato de gentamicina (Agry-gent plus ${ }^{\circledR}$ ) en dosis de $356 \mathrm{ml} \mathrm{ha}^{-1}$. Estos productos se aplicaron en dos momentos a partir de los $30 \mathrm{ddt}$.

En la etapa vegetativa se realizaron aplicaciones de insecticidas para el manejo de mosca blanca (Bemissia tabaci), los productos fueron Spiromesifen $\left(\right.$ Oberón $\left.^{\circledR}\right)$, tiametoxam+lambda cyalotrina (Engeo $\left.{ }^{\circledR}\right)$ y Thiocyclam hidrogenoxalat $\left(\right.$ Eviset $\left.^{\circledR}\right)$ en dosis de $356 \mathrm{ml} \mathrm{ha}^{-1}$ por producto, además se realizó aplicación de insecticidas botánicos como complemento de manejo de mosca blanca y el piojo del tomate (Halticus $s p$ ) a base de aceite de Azadirachtína $\left(\mathrm{Nim}^{\circledR}\right)$ en dosis de $570 \mathrm{ml} \mathrm{ha}^{-1}$ y extracto de hojas de madero negro (Gliricidia sepium (Jacq.) Kunth ex Walp., aplicando 285 litros ha ${ }^{-1}$.

Cosecha. La cosecha se realizó en cinco cortes a partir de los 84 días después del trasplante. 


\section{RESULTADOS Y DISCUSIÓN}

Diámetro ecuatorial y polar (cm). Según Gillaspy et al., 1993, los frutos presentan durante su desarrollo tres fases: desarrollo del ovario, división celular y expansión celular, que representa el crecimiento en diámetro de los frutos. El diámetro polar y ecuatorial del fruto son variables que determinan el tamaño y la forma del mismo. El tamaño del fruto varía según el material genético (Mayorga, 2004), adjudicado a cinco pares de genes (Santiago et al., 1998).

El cuadro 2 indica que el diámetro ecuatorial varía desde 3.95 a $5.58 \mathrm{~cm}$, siendo el cultivar AVTO1023 el que registra mayor diámetro, seguido de Shanty, AVTO1059, AVTO1058, AVTO1004 y AVTO1005; en cambio, el diámetro polar varía entre 4.45 y $6.95 \mathrm{~cm}$, registrándose mayores valores en los cultivares CLN3125L, Shanty y AVTO1078.

Gonzales y Laguna (2004), mencionan que los frutos pueden clasificarse como frutos grandes cuando su calibre (diámetro) es mayor a ocho centímetro, medianos entre 7.9 a $5.7 \mathrm{~cm}$ y pequeños los inferiores o iguales a $5.6 \mathrm{~cm}$. De acuerdo a esta información, los frutos de los cultivares evaluados, se clasifican como medianos y pequeños; el híbrido Shanty, presentó un calibre mediano, cultivar de buena preferencia por el consumidor nacional. Éstos cultivares no presentarían inconvenientes para su comercialización de acuerdo a este parámetro, los cuales son influenciados por factores genéticos, como lo plantea García y Hedden (1997), al indicar que el incremento en el volumen de los frutos es regulado por la fitohormona giberelina; responsable de la expansión celular, dependiendo principalmente de la naturaleza de origen, concentración y condiciones climáticas (Fos et al., 2001, citado por Ortega-Martínez, 2013).

Número de lóculos. Lóculo es la cavidad de un órgano, generalmente un fruto, esporangio o antera, que contiene semillas, esporas o granos de polen (Van Haeff, 1990), estos son formados a partir del gineceo (Rodríguez, 1998). El número de lóculo es una característica de importancia comercial (León, 2000).

En el cuadro 2 se puede apreciar que el número de lóculos varió significativamente registrándose medias entre 2.36 y 4.02 lóculos, siendo los cultivares AVTO1023, AVTO 1058 y Shanty, los que registraron mayor número. Los cultivares AVTO1023 y Shanty fueron superiores a los demás cultivares. González y Laguna, (2004) indican que el tamaño, peso y la forma del fruto del tomate están determinados por el número de lóculos, relación registrada en los cultivares, ya que a mayor número de lóculos, mayor es el peso y el diámetro ecuatorial.

Grados Brix $\left({ }^{\circ} \mathbf{B x}\right)$. Los grados brix indican el contenido de sólidos solubles contenidos en el jugo de tomate y determinan una relación directa de las cantidades de materia prima a obtenerse para la industria (Alemán y Pedroza, 1991). Valores mayores o iguales a cuatro grados Brix, es considerado bueno (Santiago et al., 1998).
En el cuadro 2 se observa valores entre 4.08 y 5.08 ${ }^{\circ} \mathrm{Bx}$. Los cultivares AVTO1059, AVTO1008 y CLN3125L registraron mayores valores en grados Brix. Aguayo y Artés (2004) consideran que de 4.5 a 5.5 grados Brix son valores óptimos en cuanto a calidad de los frutos de tomate, ubicando a los cultivares en esta clasificación, en cambio González y Laguna (2004), plantean que valores entre 3.5 y $7{ }^{\circ} \mathrm{Bx}$ es un parámetro admisible para la industrialización, por lo que todos los cultivares son aceptables para la industria y el comercio.

Cuadro 2. Diámetro ecuatorial, diámetro polar, número de lóculo y grados Brix de 12 cultivares de tomates evaluados en casa malla

\begin{tabular}{lcccc}
\hline Cultivares & $\begin{array}{c}\text { Diámetro } \\
\text { ecuatorial }\end{array}$ & $\begin{array}{c}\text { Diámetro } \\
\text { polar }\end{array}$ & $\begin{array}{c}\text { Número de } \\
\text { lóculos }\end{array}$ & $\begin{array}{c}\text { Grados } \\
\text { Brix }\end{array}$ \\
\hline CLN3125L & 4.08 & 6.95 & 2.38 & 4.89 \\
Shanty & 4.84 & 6.85 & 3.42 & 4.54 \\
AVTO1078 & 4.05 & 6.50 & 2.39 & 4.80 \\
AVTO1005 & 4.49 & 5.90 & 2.93 & 4.65 \\
AVTO1032 & 4.19 & 5.85 & 2.70 & 4.66 \\
AVTO1023 & 5.58 & 5.75 & 4.02 & 4.60 \\
AVTO1008 & 4.07 & 5.70 & 2.58 & 5.07 \\
AVTO1004 & 4.49 & 5.65 & 3.05 & 4.73 \\
AVTO1059 & 4.70 & 4.85 & 3.29 & 5.08 \\
AVTO1058 & 4.66 & 4.75 & 3.91 & 4.61 \\
1x10 & 3.95 & 4.65 & 2.36 & 4.66 \\
AVTO1143 & 4.11 & 4.45 & 3.21 & 4.08 \\
DMS (95\%) & 0.44 & 0.65 & 0.68 & 0.59 \\
\hline
\end{tabular}

Forma, color de fruto e intensidad de color en frutos maduros. La forma de los frutos es un criterio que con frecuencia permite distinguir entre cultivares de una misma especie. Generalmente, el consumidor exige un producto de una determinada forma y rechaza los ejemplares que no la poseen (Willis et al., 1999). El color representa una medida de calidad y en muchas ocasiones es la más importante a considerar al momento de la comercialización, también el color de la epidermis, es un buen indicador del estado de madurez de los frutos (Clemente, 2010).

Los caracteres cualitativos de forma, color e intensidad de color a la madurez, mostraron diferencias entre los cultivares. La forma de fruto que predominó fue el redondo-alargado, seguido de la forma cilíndrica (oblongo-alargado) y de la forma redondeada. Todos los cultivares presentaron color rojo y la intensidad de color a la madurez predominante fue la intermedio (cuadro 3). Cantín (2009), citado por Agudelo (2011), afirma que las variables diámetro ecuatorial y diámetro polar, son indicadores del tamaño y forma de los frutos, este mismo autor asegura que el color de los frutos se debe a los contenidos de carotenoides contenidos en cada cultivar. 
Cuadro 3. Clasificación de los cultivares de tomate de acuerdo a la forma del fruto, color e intensidad de color a madurez

\begin{tabular}{lll}
\hline \multicolumn{1}{c}{ Cultivares } & \multicolumn{1}{c}{ Forma del fruto } & \multicolumn{1}{c}{$\begin{array}{c}\text { Intensidad } \\
\text { de color }\end{array}$} \\
\hline AVTO1004 & Cilíndrico (oblongo-alargado) & Intermedio \\
AVTO1058 & Redondo-alargado & Intermedio \\
AVTO1032 & Redondo-alargado & Intermedio \\
AVTO1008 & Cilíndrico (oblongo-alargado) & Intermedio \\
AVTO1078 & Cilíndrico (oblongo-alargado) & Intermedio \\
AVTO1059 & Redondeado & Intenso \\
1x10 & Redondo-alargado & Intenso \\
AVTO1005 & Redondo-alargado & Poca \\
Shanty & Cilíndrico (oblongo-alargado) & Poca \\
AVTO1023 & Redondo-alargado & Poca \\
AVTO1143 & Redondeado & Poca \\
\hline
\end{tabular}

Peso del fruto (g). El fruto de tomate es una baya bi o plurilocular que puede alcanzar un peso promedio de 600 gramos. El peso del fruto está determinado por la relación entre la potencia de la fuente de nutrientes y la potencia de la demanda de nutrientes durante el periodo de crecimiento del fruto (Santiago et al., 1998).

En el cuadro 4 se indica que el peso de fruto vario entre 53.96 y 110.3 g, siendo los cultivares AVTO1023 y Shanty los que registraron mayor peso. Las diferencias en peso de fruto se deben a la constitución genética propia de cada cultivar, sin embargo, es un carácter poco estable en el cultivo de tomate, principalmente cuando es influenciado por el ambiente (Santiaguillo et al., 2004).

El INTA (2012) reporta que la variedad Shanty produce frutos con pesos entre 45 y $122 \mathrm{~g}$. Los cultivares presentaron valores entre este rango, por lo que el peso registrado no sería un inconveniente para la comercialización de estos cultivares. Huerres y Carballo (1998), plantean que los tomates aptos para el aprovechamiento industrial, por lo general alcanzan pesos promedios no mayores a $150 \mathrm{~g}$. En el mercado nacional se observa que los consumidores utilizan tomate industrial para consumo fresco, por tanto estos cultivares podrían ser utilizados para uso industrial y para consumo fresco.

Número de frutos por planta. La cantidad de frutos por planta está determinado por el número de flores que son fecundadas y alcanzan a desarrollar fruto (Santiago et al., 1998), así como de la intensidad en la síntesis de fotoasimilados (Bertin, 1995).

Los cultivares presentaron entre 8 y 44 frutos por planta obteniendo mayor número de frutos los cultivares AVTO1032, CLN3125L y AVTO1059 (cuadro 4). El INTA (2012) reporta que la variedad Shanty produce entre 35 y 45 frutos por planta; al hacer la comparación, encontramos que la mayoría se ubican por debajo de este rango, igualado solo por el cultivar AVTO1032.
Rendimiento $\mathrm{kg} \mathrm{ha}^{-1}$ ) parcela $^{-1}$. El rendimiento de un cultivo está determinado por su genética, el manejo, condiciones del suelo y condiciones climáticas. Los mayores rendimientos fueron obtenidos por los cultivares AVTO 1023, CLN3125L, AVTO1058, AVTO1059 Y AVTO1032, sin embargo, todos los cultivares a excepción de Shanty y AVTO1143 superan la producción promedio nacional que es de $25200 \mathrm{~kg} \mathrm{ha}^{-1}$, obtenida en cultivos a campo abierto (MAGFOR, 2012b).

Para poder analizar el rendimiento de una planta es necesario el estudio de sus componentes de rendimiento. En el cultivo de tomate los principales componentes del rendimiento son, el número de frutos por planta y el peso de fruto. Así mismo el rendimiento involucra procesos fisiológicos relacionados con el crecimiento vegetativo y reproductivo del cultivo, y está fuertemente influenciado por la relación fuente-demanda en diferentes fases del ciclo de vida de la planta (Wereing y Patrick, 1975).

Podemos afirmar, que el rendimiento de los cultivares estuvo influenciado por su potencial genético, ubicándose por debajo de los rendimientos reportados por Jaramillo at al., (2013), que oscilan entre 100 y 150 tn ha-1 $^{-1} 100000$ y 150 $000 \mathrm{~kg} \mathrm{ha}^{-1}$ ) como se indica en el cuadro 4. Sin embargo en Nicaragua se obtienen rendimientos promedios de 17 a $25 \mathrm{t}$ / ha (INTA, 2002).

Los rendimientos obtenidos en este estudio pudieron estar influidos por problemas fitosanitarios ya que según Bolaños 2001, este factor a influido mucho en los últimos años, debido principalmente a la alta incidencia de patógenos de suelo y a virosis transmitida por la mosca blanca (Bemisia tabaci)

Cuadro 4. Pesos de fruto, frutos por planta y rendimiento según cultivar

\begin{tabular}{cccc}
\hline Cultivar & $\begin{array}{c}\text { Peso de fruto } \\
(\mathrm{g})\end{array}$ & $\begin{array}{c}\text { Frutos por } \\
\text { planta }\end{array}$ & $\begin{array}{c}\text { Rendimiento } \\
\left(\mathrm{kg} \mathrm{ha}^{-1}\right)\end{array}$ \\
\hline AVTO1023 & 110.3 & 8.4 & 82250 \\
CLN3125L & 80.7 & 33.7 & 66350 \\
AVTO1032 & 73.9 & 44.1 & 63000 \\
AVTO1059 & 75.6 & 33.5 & 61250 \\
AVTO1058 & 78.1 & 30.3 & 51100 \\
AVTO1078 & 74.4 & 29.7 & 47250 \\
AVTO1005 & 83.5 & 24.0 & 44850 \\
AVTO1008 & 72.7 & 23.3 & 39350 \\
$1 \times 10$ & 54.0 & 22.8 & 30500 \\
AVTO1004 & 80.1 & 13.9 & 26850 \\
Shanty & 100.2 & 32.4 & 24250 \\
AVTO1143 & 56.3 & 16.2 & 22000 \\
\hline DMS (95\%) & 17.3 & 15.8 & \\
\hline
\end{tabular}




\section{CONCLUSIONES}

Las formas de frutos más comunes de los cultivares fue la cilíndrico (oblongo-alargado) y redondo-alargado.

Los cultivares que mostraron mejor rendimiento y adaptabilidad fueron CLN3125L, AVTO1032, AVTO1059, AVTO1058, AVTO1078 y AVTO1005.
En base al rendimiento, los cultivares promisorios para continuar con evaluaciones en condiciones de ambiente protegido son, CLN3125L, AVTO1032, AVTO1059, AVTO1058, AVTO1078, AVTO1005.

\section{REFERENCIAS BIBLIOGRÁFICAS}

Aguayo Giménez, E.; Artés Calero, F. 2004. Elaboración del tomate mínimamente procesado en fresco. Ediciones de Horticultura S.L., Reus, ES.

Alemán M. G.; Pedroza H, P. 1991. Manejo Integrado de Plagas. Artículos N 50. San José, CR. 100 p.

Agudelo, A, A; Ceballos, A, N; Orozco, F, J; 2011. Caracterización morfológica del tomate tipo cereza (solanum lycopersicum linnaeus) universidad de Caldas, Colombia. Fuente original: Cantín, C. 2009. Estudio agronómico y de la calidad del fruto del melocotonero (P. persica (1.) batsch) en diferentes poblaciones de mejora para la selección de nuevos cultivares. Universidad de Zaragoza. Zaragoza, Aragón. España. Agron. 19(2): 44 - 53. (en linea). Consultado 24 nov. 2013. Disponible en http://www. cabi.org/isc/FullTextPDF/2013/20133297549.pdf

Bertin, N. 1995. Competition for assimilates and fruit position affect fruit set in indeterminate greenhouse tomato. (en línea). Consultado 13 ene. 2014. Disponible en http://www.ncbi.nlm.nih.gov/pmc/articles/PMC3023665/pdf/75-1 55.pdf

Bolaños, HA. 2001. Introducción a la holericultura. 2ed. San José, Costa Rica. Editorial Universidad Estatal a Distancia.

Carrillo, J.C.; Jiménez, F.; Ruiz, J.; Díaz, G.; Sánchez, P.; Perales, C.; Arellanes, A. 2003. Evaluación de densidades de siembra en tomate (Lycopersicon esculentum Mill) en Invernadero. AGRONOMIA MESOAMERICANA. 14(1):85-88 p.

Clemente Lezama, N. 2010. Calidad postcosecha de tomate (Solanum lycopersicum L.) producido en agua residual y de pozo en hidroponía y suelo. Tesis M.Sc. en Horticultura. UACh. Chapingo, MX. 73 p.

EDA (Entrenamiento y Desarrollo de Agricultores). 2006. Boletín de mercadeo: "conocer su producto tomate". FHIA (Fundación Hondureña de Ibvestigación Agropecuaria). HN. 94 p.

Fos, M, Proaño, K; Nuez, F; García, MJ. 2001. Role of gibber - ellins in parthenocarpic fruit development induced by the genetic system pat-3/pat-4 in tomato. Physiol. Plant. 111: 545-550. Fuente original: Ortega-Martínez LD; Ocampo Mendoza, J; Martínez Valenzuela, C; Pérez Serrano, A; Sánchez Olarte, J. 2013. Efecto de las giberelinas sobre el crecimiento y calidad de plántulas de tomate. Biotecnia XV(3):56. (en línea). Consultado 27 nov. 2013. Disponible en http://www.biotecnia.uson.mx/ revistas/articulos/24-Articulo\%209.pdf

García Martínez, J.L. y P. Hedden. 1997. Gibberellins and fruit development. Eds. Tomas Barberan, F.A. y R.J. Robins. Phytochemistry of fruit and vegetables. Oxford Sci. Publications, Heidelberg. 263-285.

Gillaspy, G.; H. Ben David.; Gruissem, W. 1993. Fruits: a developmental perspective. Plant Cell. 5:1439-1451.

González Madrigal, F.M. 2013. Evaluación preliminar de 27 genotipos de tomate (Licopersicum sculentum Mill) tolerantes al complejo mosca blanca (Bemisia tabaci Gennadius (Hemíptera: Aleyrodidae)) - Geminivirus, Tisma, Masaya, postrera, 2010. Tesis Ing. Agrom. CENIDA, UNA. Managua, NI. 29 p.

González Urrutia, O.E.; Laguna Laguna, J.L. 2004. Evaluación del comportamiento agronómico de once cultivares de tomate (Lycopersicon esculentum Mill), bajo el manejo del productor en el valle de Sébaco, Matagalpa. (en línea). Managua, NI, UNA. Consultado 21 ene. 2014. Disponible en http://cenida.una.edu.ni/Tesis/tnf30g643.pdf

Huerres, C.; N. Carballo. 1988. Cultivo del tomate y pimiento. Pueblo y educación. La Habana, CU. 30 p.

INETER (Instituto Nicaragüense de Estudios Territoriales). 2013. Datos meteorológicos y geográficos. Managua, NI.

INTA (Instituto Nicaragüense de Tecnología Agropecuaria). 2012. Cultivo del tomate. ed. 22. Managua, NI. Editorial Inpasa. 1-17 p.

INTA (Instituto Nicaragüense de Tecnología Agropecuaria). 2002. Cultivemos tomate con Menos Riesgo. Sanabria; J Mercado. 1 ed. Managua, NI. 37 p

Jaramillo Noreña, JE; Sánchez León, GD; Rodríguez, VP; Aguilar Aguilar, PA; Gil Vallejo, LF; Hío, JC; Pinzón Perdomo, LM; García Muñoz, MC; Quevedo Garzón, D; Zapata Cuartas, MÁ; Restrepo, JF; Guzmán Arroyave, M. 2013. Tecnología para el cultivo de tomate bajo condiciones protegidas. CORPOICA, Bogotá, CO. 482 p.

Jaramillo, J; Rodríguez, VP; Guzmán, M; Zapata, M; Rengifo, T. 2007. Manual técnico: Buenas prácticas agrícolas en la producción de tomate bajo condiciones protegidas. CORPOICA, MANA, Gobierno de Antioquia, FAO. Antioquia, CO. 331 p.

Jaramillo Noreña, J; Rodríguez, VP; Guzmán, M; Zapata, MA. 2006. El cultivo de tomate bajo invernadero (Lycompersicon esculentum Mill). CORPOICA. Antioquia, CO. 48 p.

León, J. 2000. Botánica de los cultivos tropicales. Tercera Edición. Editorial Agro América San José CR. 319-320 p.

López Benítez, A.; Borrego Escalante, F.; Zamora Villa; V.M.; Guerra Zitlalapa, L. 2012. Estimación de Aptitud Combinatoria General y Aptitud Combinatoria Específica en Siete Líneas de Tomate (Solanum lycopersicum L.). (en línea). Saltillo, MX. Consultado 22 set. 2013. Disponible en http://www.uaaan.mx/agraria/attachments/article/74/Revista_Agraria_Vol(9)_No(3)_ A2.pdf

MAGFOR (Ministerio Agropecuario Forestal). 2012a. Beneficios del programa para la producción de solanáceas en el país. Managua, NI. 2 p. 
2012b. Plan de acción regional para el manejo de las moscas. Managua, NI. 1 p.

Mayorga Suchite, A.S. 2004. Evaluación agronómica de ocho híbridos de tomate (Lycopersicum esculentum L.) en dos localidades de Zacapa. (en línea). Chiquimula, GA. USAC. Consultado 21 ene. 2014. Disponible en http://cunori.edu.gt/descargas/EVALUACIN_AGRONOMICA_DE_OCHO_HIBRIDOS_DE_TOMATE_EN_DOS_LOCALIDADES_DE_ZACAPA.pdf

Rayo, M. 2001. Caracterización biológica transmitido por mosca blanca (Bemisia tabaci Genn.) en el cultivo del tomate (Lycopersicum esculentun Mill) en el Municipio de Santa Lucía, Boaco y la evaluación de diferentes materiales de tomate sometidos a inoculación artificial y natural antes el complejo mosca blanca-Geminivirus. Managua, NI. 1-4 p.

Rodríguez, 1998. Cultivo del Tomate. Edición 22, Instituto Nicaragüense de Tecnología Agropecuaria, Editorial Inpasa, Managua, NI. $32 \mathrm{p}$.

Santiago, J.; Mendoza, M.; Borrego, F. 1998. Evaluación de tomate (Lycopersicon esculentum, MILL) en invernaderos: criterios fenológicos y fisiológicos. Agronomía Mesoamericana. 9(1):59-65.

Santiaguillo Hernández, J.F.; Cervantes Santana, T.; Peña Lomelí, A. 2004. Selection for fruit yield and quality from plant x plant crosses between husk tomato varieties. Rev. Fitotec. Mex. 27 (1):85 - 91.

Smith, AF. 1994. The tomato in America: early history, culture, and cookery. University of South Carolina Press, Columbia, S.C, USA.

Van Haeff, J N. 1990. Tomates. Segunda edición. Trillas. México. 54 p.

Willis R, H.H.; Lee, T.H.; McGlasson, W.B.; Hall, E.G.; Graham, D. 1999. Introducción a la fisiología y manipulación de las frutas, hortalizas y plantas ornamentales. 2da. Ed. Acribia. Zaragoza, ES. 240 p.

WEREING, P. E; PATRICK J. 1975. Source-sink relations and partition of assimilates. In J. P. Cooper Celd, photosynthesis and productivity in differents environments. Cambridge Univ. Press. p. 481-499. 